\title{
VALIDATION OF A LOW FIDELITY CATENARY MODEL DEVELOPED USING A NOVEL OPTIMIZATION ALGORITHM
}

\author{
Rosalie Morin ${ }^{1}$, Ben Shiff ${ }^{1}$, Kee Seung Oh$^{1}$, Stephen William Knox Roper ${ }^{1}$, Il Yong Kim ${ }^{1 *}$ \\ ${ }^{1}$ Department of Mechanical and Materials Engineering, Queen's University, Kingston, Canada \\ *kimiy@queensu.ca
}

\begin{abstract}
Contact wire pre-sag directly impacts the current quality collection in a high-speed railway catenary. Due to this, the initial configuration of the catenary geometry plays an important role on the dynamic performance of the railway. Therefore, accurately representing the initial equilibrium state of the catenary based on specific design requirements is crucial to obtain accurate dynamic results. Despite its importance, there are only a few publications in this area that present methods that can accommodate desired amount of presag in the contact wire and are computationally efficient. The goal of this paper is to present a catenary system that has been modelled using a novel optimization method and validate its dynamic response from its interaction with a pantograph system against the reference model results in BS EN 50318. The novel optimization methodology presented in this paper employs a gradient-based algorithm with a modified finite difference method to solve the initial equilibrium geometry of the catenary. The pantograph and catenary systems are modelled using a commercial finite element software and the post-processing of the results is done using in-house code. A penalty contact-force model is used to represent the contact behavior between the pantograph-catenary system and a threestep simulation procedure is used to achieve better convergence of results. The results from the simulation demonstrated good accordance with the reference model results in BS EN 50318.
\end{abstract}

Keywords- catenary modelling; initial equilibrium problem; pantograph-catenary dynamic interaction; finite element analysis

\section{INTRODUCTION}

In modern railways, catenary systems are used to transmit power to the train by means of contacting the pantograph. Uniform contact force throughout the interaction between the pantograph and catenary system is critical in order to maintain high current collection quality. Large contact forces increase friction and wear on pantograph collector strips, while low forces can lead to sparking and wear due to electrical arcing. This variation of the contact force between the pantograph and catenary system are mainly a result of stiffness variations and wave propagations along the catenary [1]. Studies have also shown that the pre-sag of the contact wire influences the

\footnotetext{
NSERC, SNC-Lavalin
}

dynamic interaction of the pantograph and catenary system [1]. Therefore, properly modelling the initial equilibrium state of the catenary is important to obtain an accurate dynamic response to ensure the train of a light rail system receives enough power.

The catenary system is a complex system of wires with low bending stiffness that displays strong nonlinear geometric behavior under high tensile forces. Due to this behavior, there's a challenge in modelling the initial configuration of the unstressed catenary system as the undeformed reference geometry is unknown. This is known as the initial equilibrium problem [3]. Several approaches have been developed to solve this problem namely, analytical methods and finite element (FE)-based approaches [3-7]. However, not much work has been done in the development of a method that can be adapted to several different catenary configurations and is computationally efficient [8]. In this paper, a novel FE-based methodology is utilized to model the undeformed catenary geometry that will result in the desired deformed geometry after both gravity and tension are applied to the system [9]. This method employs a gradient-based algorithm and uses a modified finite difference method (FDM) for sensitivity analysis. It can accommodate irregular catenary geometries as it enables the user to specify known geometrical points, such as the amount of desired pre-sag in the contact wire, after both tension and gravity are applied to the system. The catenary geometry is then used for transient analysis where all required outputs are determined.

The current modelling and simulation methodology proposed in this paper leverages ANSYS commercial finite element software and in-house code. The dynamic interaction between the catenary and pantograph models are validated in accordance to standard BS EN 50318. This standard presents a method for validating simulations of the dynamic interaction of a pantograph-catenary system [2]. The method recommends two stages of validation, referred to as Validation 1 and Validation 2. For Validation 1, the simulation results are validated against a reference model. The parameters, such as mean contact force and percent contact loss, must all fall within the acceptable range of results. Once the results satisfy these requirements, the simulation must be validated against real line data for Validation 2. The simulation results for the model 
presented in this paper have been validated against the reference model from the standard.

This paper is organized as follows: Section II describes the modelling approach for the low fidelity pantograph and catenary system. Section III introduces the approach for representing the dynamic interaction between the catenarypantograph systems, as well the post-processing method for filtering the simulation data. Section IV presents and discusses the results obtained from the simulation. Finally, Section V discusses main conclusions and future works for the current work.

\section{MODELLING THE CATENARY AND PANTOGRAPH SYSTEMS}

\section{A. Low fidelity catenary model}

\section{1) Catenary reference model}

The geometry and features incorporated in the catenary model are based on the reference model found in BS EN 50318. The catenary reference model is defined to have a messenger wire, contact wire, nine droppers, and two steady arms per span. Ten identical spans are incorporated in the catenary model at a +/- $0.2 \mathrm{~m}$ stagger. However, the catenary model developed does not incorporate stagger because only two-dimensional analysis between the pantograph and catenary is considered. Additional parameters such as span length, encumbrance, and dropper position are also defined by the standard and presented in Fig. 1 and TABLE I.

\section{2) Finite element modeling of the catenary system}

The contact and messenger wires are represented with Euler-Bernoulli beam elements, which correspond to BEAM188 in the commercial software used. Typically, copper alloys are used for the wires in the catenary system. For this catenary model, Copper Magnesium ( $\mathrm{CuMg}$ ) Bronze is assumed for both the messenger and contact as it is commonly used in high-performance railway lines [10]. The cross section of the wires is assumed as circular and a mesh density of 30 elements is used between each dropper to accurately capture the wave propagation of the contact and messenger wires. The mesh density was determined through a mesh convergence study. The steady arms are also modelled using BEAM188 elements with a circular cross section and material properties assumed to be that of mild steel. The droppers, which connect the contact and messenger wires, are modelled using unidirectional spring elements COMBIN39. The spring stiffness is defined as $100,000 \mathrm{~N} / \mathrm{m}$ in tension and $0 \mathrm{~N} / \mathrm{m}$ in compression. Additionally, the mass of the droppers and the clamps used to attach the droppers to the messenger and contact wires are taken to be zero in the reference model. Therefore, no additional lumped masses have been included to represent these features. For Validation 1, the standard also specifies zero damping in the catenary system, thus no damping method is used to represent the dissipation of energy in the system.
TABLE I. CATENARY PARAMETERS FROM THE REFERENCE MODEL

\begin{tabular}{|l|c|c|}
\hline \multirow{2}{*}{\multicolumn{1}{|c|}{ Catenary Data }} & \multicolumn{2}{c|}{ Parameters } \\
\cline { 2 - 3 } & Value & Units \\
\hline Contact wire tension & 20000 & $\mathrm{~N}$ \\
\hline Contact wire linear density & 1.35 & $\mathrm{~kg} / \mathrm{m}$ \\
\hline Messenger wire tension & 16000 & $\mathrm{~N}$ \\
\hline Messenger wire line density & 1.07 & $\mathrm{~kg} / \mathrm{m}$ \\
\hline Spring constant of droppers & 100000 & $\mathrm{~N} / \mathrm{m}$ \\
\hline $\begin{array}{l}\text { Mass of links between wires } \\
\text { and droppers (clamps) }\end{array}$ & 0 & $\mathrm{~kg}$ \\
\hline Steady arm line density & 1.0 & $\mathrm{~kg} / \mathrm{m}$ \\
\hline
\end{tabular}

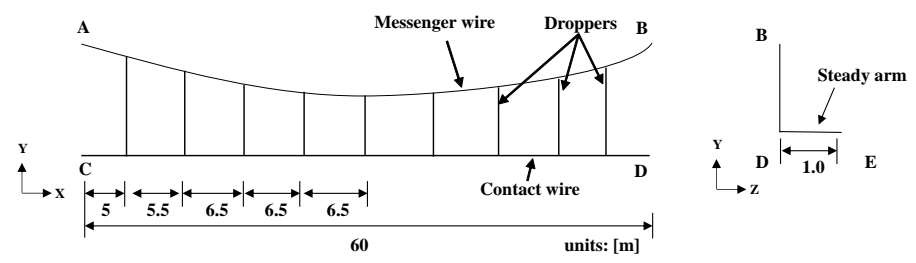

Figure 1. This figure details the encumbrance, distance between droppers, and span length for one span in the reference model. Note: the span is symmetrical, thus only the values for the first five droppers are shown.

\section{3) Initial equilibrium of the catenary system}

The initial equilibrium state of the catenary is based on the reference model detailed in BS EN 50318 and Fig 1. Therefore, the desired deformed geometry is known, while the undeformed geometry is unknown. This poses a challenge, as the undeformed geometry must be defined in ANSYS to run the simulation. To solve this problem, a novel optimization methodology is utilized to determine the initial undeformed geometry that will result in the desired deformed geometry after both gravity and tension are applied to the system. The FE-based method minimizes the error between the initial deformed geometry obtained with ANSYS and the target points from the deformed reference geometry [9]. This section discusses the unknown user-specified target points, such as the $y$-coordinate points for nodes connecting the droppers to the contact and messenger wires, and briefly presents the optimization algorithm.

To obtain the deformed reference geometry, the lengths of each dropper can be manipulated. Knowledge on the desired amount of contact wire pre-sag in a catenary system is helpful, as it is function of dropper length [1]. The reference model is stated to have zero pre-sag; therefore, after applying the gravitational and tensile forces on the catenary, the resulting deformed geometry must match the reference geometry outlined in Fig. 1. The lengths of the droppers are calculated using an analytical method that assumes the system to be in static equilibrium [1]. This method assumes uniform linear density within the wires, symmetry about the center of the span, and equal encumbrance and height at the steady arms. Additionally, concentrated masses such as insulators and clamps are not considered. The reader is referred to the source for access to the full equations [1]. The calculated lengths for each dropper are shown in Fig. 2 and are used as target points in the optimization algorithm. 


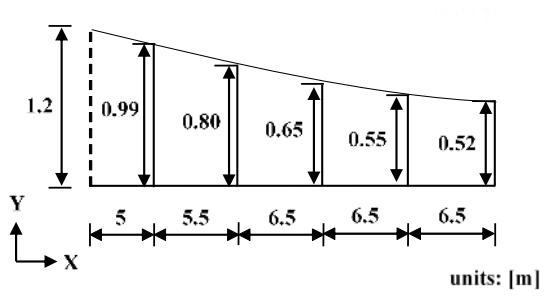

Figure 2. Catenary reference model with the dropper lengths calculated for the first five of droppers of one span to achieve zero pre-sag.

The optimization method minimizes the half mean squared error of the nodal $x$ and $y$ coordinates $(\boldsymbol{\chi})$ for nodes connecting the dropper to the contact and messenger wires, as well as the nodes located on mast points. Error is considered as the difference between the target nodal coordinate $\left(\boldsymbol{\chi}^{\text {target }}\right)$ and current nodal coordinate $(\bar{\chi})$. In the case of no pre-sag, the vertical target coordinate points for the dropper and contact wire connections is zero, and the vertical target point for the dropper and messenger wire connections is its respective dropper length. The optimization function defined in (1) determines the initial geometry required to attain the deformed target geometry within a user defined tolerance. With this method the initial geometry can be determined for any combination of pre-sag and tensile forces, expanding the scope of problems that can be analyzed using the methods outlined in this paper. To solve the optimization problem in (1), the Broyden-Fletcher-Goldfarb-Shanno algorithm is used by introducing SciPy which is a free and open-source Python library. The reader is referred to the source for further details about the algorithm [9].

$$
L(\boldsymbol{\chi})=\frac{1}{2} \mathbf{1} \cdot\left[\left(\boldsymbol{\chi}^{\mathrm{target}}-\overline{\boldsymbol{\chi}}\right) \circ\left(\boldsymbol{\chi}^{\mathrm{target}}-\bar{\chi}\right)\right]
$$

\section{B. Low fidelity pantograph model}

\section{1) Pantograph reference model}

According to the reference model in BS EN 50318, the pantograph is to be modeled as a discrete mass-spring damper system (as shown in Fig. 3) that does not consider bump stops, frictional forces associated with contact, or the effect of aerodynamic forces. The pantograph uplift force, $F_{l}$, is represented as a constant $120 \mathrm{~N}$ force acting on the mass, $m_{2}$. The pantograph is modelled in two-dimensions; considering the horizontal $(x)$ and vertical ( $y$ ) influence of the pantograph as it moves along the contact wire.

\section{2) Finite element modeling of the pantograph}

To represent the simplified pantograph model in ANSYS, both lumped masses are modelled with MASS21 elements, while the spring-damper systems are modelled with COMBIN14 elements. These elements are defined in accordance with the data provided in the reference model.

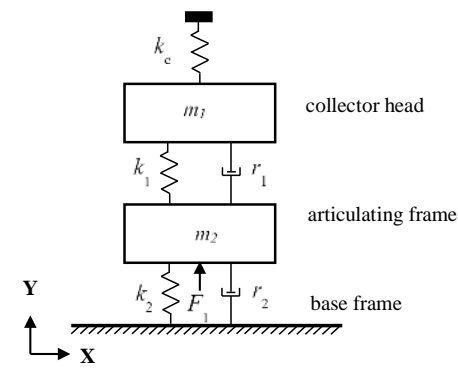

Figure 3. Pantograph model represented as a discrete mass-spring damper system. All parameters shown are defined in BS EN 50318.

TABLE II. PANTOGRAPH PARAMETERS FROM THE REFERENCE MODEL

\begin{tabular}{|c|c|c|}
\hline \multirow{2}{*}{ Pantograph Data } & \multicolumn{2}{|c|}{ Description } \\
\cline { 2 - 3 } & Parameter [unit] & Value \\
\hline \multirow{2}{*}{ Lumped mass } & $\mathrm{m}_{1}[\mathrm{~kg}]$ & 7.2 \\
\cline { 2 - 3 } & $\mathrm{m}_{2}[\mathrm{~kg}]$ & 15 \\
\hline \multirow{2}{*}{ Lumped spring } & $\mathrm{k}_{1}[\mathrm{~N} / \mathrm{m}]$ & 4200 \\
\cline { 2 - 3 } & $\mathrm{k}_{2}[\mathrm{~N} / \mathrm{m}]$ & 50 \\
\hline Contact spring & $\mathrm{k}_{\mathrm{c}}[\mathrm{N} / \mathrm{m}]$ & 50000 \\
\hline \multirow{2}{*}{ Lumped damper } & $\mathrm{r}_{1}[\mathrm{Ns} / \mathrm{m}]$ & 10 \\
\cline { 2 - 3 } & $\mathrm{r}_{2}[\mathrm{Ns} / \mathrm{m}]$ & 90 \\
\hline Static/uplift force & $\mathrm{F}_{1}[\mathrm{~N}]$ & 120 \\
\hline
\end{tabular}

A physical representation of the pantograph collector strip is also incorporated in the pantograph model to properly define the line-line contact at the interface of the pantograph collector strip and the contact wire. The collector strip is modelled with BEAM188 elements with a cross-section assumed to be circular.

\section{PANTOGRAPH-CATENARY SYSTEM INTERACTION}

\section{A. Contact modelling}

CONTA176 and TARGE170 elements are used to represent line-to-line contact between the contact and target points. Key options have been used to define the contact algorithm, contact model, and behavior of the contact surface to represent the dynamic interaction between both components. A penalty contact-force based model is selected to represent the sliding contact between the pantograph-catenary systems. The penalty method uses a contact spring stiffness to establish a relationship between both contact lines [11]. This method is widely used in existing pantograph-catenary software and is selected because the contact spring stiffness is defined by the standard as $50,000 \mathrm{~N} / \mathrm{m}$ [12]. Furthermore, to capture the sliding contact between the crossing beams of the pantographcatenary system, the normal component of the contact force is considered, and the effect of friction is neglected.

\section{B. Three-step simulation procedure}

Static equilibrium of the system must be achieved before the pantograph begins to move horizontally across the catenary system in the $x$ direction. As a result, a three-step simulation procedure is adopted and detailed in the following paragraphs. Note, the boundary conditions for steps 1 and 2 are defined 

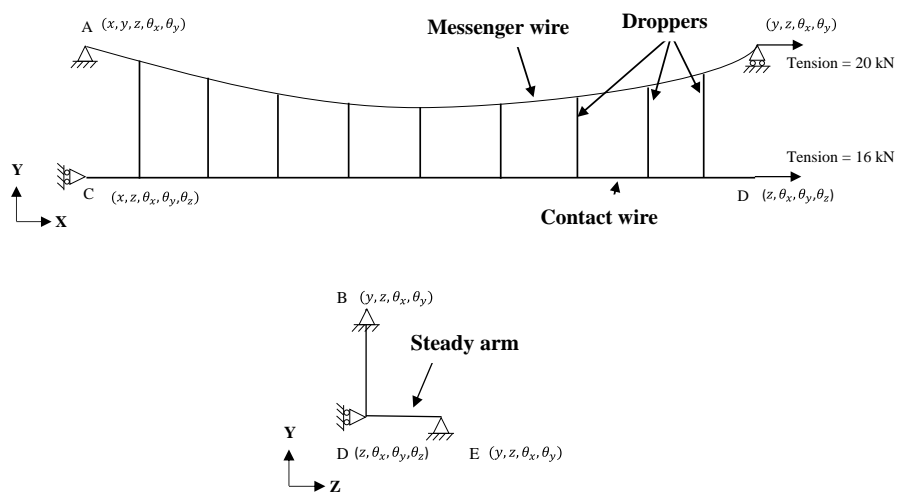

Figure 4. The boundary conditions on the catenary model for static analysis

with reference to BS EN 50318. These constraints are shown in Fig. 4. Furthermore, as two-dimensional analysis is of interest, nodes are constrained against displacements in the $z$ (lateral) direction and rotations about $\theta_{x}$ (horizontal) and $\theta_{y}$ (vertical).

Step 1 and 2 (Static): Tension and gravity is applied to all components of the catenary to allow the system to reach equilibrium prior to the interaction with the pantograph. To enable this, the collector head of the pantograph is initialized at a small distance below the contact wire. To model gravity and tension in the catenary model, accelerations equal to the gravitational constant are applied in the $y$ direction to all the nodes in the system and constant tensioning forces of $20 \mathrm{kN}$ and $16 \mathrm{kN}$ are applied horizontally to the contact and messenger wire ends. In step 2, the uplift force is applied on the articulating frame of the pantograph. This step closes the initial gap between the collector head and the contact wire and determines the equilibrium position of the system prior to movement of the pantograph.

Step 3 (Transient): Velocities equal to the speed of the train specified by the standard are applied to all the nodes on the pantograph in the $\mathrm{x}$ direction. The duration of the load step is set to match the amount of time required to meet the end of the line section. The Newmark method is used to solve the FE equations of motion to compute the contact force at each nodal position.

\section{Signal processing}

According to the BS EN 50318, the frequency range of interest for the numerical results is $0 \mathrm{~Hz}$ to $20 \mathrm{~Hz}$. For this model, a low pass filtering method is required to filter out the high frequency oscillations resulting from the use of the penalty method [13]. The filtering is performed using a $6^{\text {th }}$ order Butterworth low pass filter. This filter has been selected because the frequency response from this filter is maximally flat (unity in the passband and zero in the stopband) and has monotonic amplitude response for both passband and stopband [14].

\section{SIMULATION RESUltS}

TABLE III. SIMULATION RESULTS

\begin{tabular}{|c|c|c|}
\hline \multirow{2}{*}{$\begin{array}{l}\text { Range of results for } \\
\text { validation } 1\end{array}$} & \multicolumn{2}{|c|}{ Speed $250[\mathrm{~km} / \mathrm{h}]$} \\
\hline & $\begin{array}{c}\text { Reference model (BS } \\
\text { EN 50318:2002) }\end{array}$ & Model \\
\hline Mean contact force $[\mathrm{N}]$ & 110 to 120 & 113.18 \\
\hline Standard deviation $[\mathrm{N}]$ & 26 to 31 & 26.54 \\
\hline Statistical maximum [N] & 190 to 210 & 192.8 \\
\hline Statistical minimum $[\mathrm{N}]$ & 20 to 40 & 33.57 \\
\hline Actual maximum $[\mathrm{N}]$ & 175 to 210 & 175.9 \\
\hline Actual minimum $[\mathrm{N}]$ & 50 to 75 & 55.24 \\
\hline $\begin{array}{l}\text { Minimum uplift at } \\
\text { support }[\mathrm{mm}]\end{array}$ & 48 to 55 & 44.71 \\
\hline Percent contact loss [\%] & 0 & 0 \\
\hline
\end{tabular}

The pantograph-catenary interaction is evaluated for pantograph speeds for $250 \mathrm{~km} / \mathrm{h}$, with results being processed for spans 5 and 6 at a cut-off frequency of $20 \mathrm{~Hz}$. The numerical results are shown in table IV. At speeds of 250 $\mathrm{km} / \mathrm{h}$, the simulation results from the model demonstrate that all, but one output, falls within the acceptable range of results for Validation 1. The minimum uplift at support is short by 3.3 $\mathrm{mm}$. This may be a result of excluding stagger of the line in the catenary model. Another reason for this may be due to the stiffness variations throughout the span: stiffness is highest at the supports due to the steady arms and lowest at the center of the spans [1].

\section{CONCLUSION}

This paper presents an overview of modeling a pantographcatenary system in accordance with BS EN 50318 standard requirements. The finite element modeling process for the pantograph and catenary systems is presented with the application of a newly developed methodology for defining the initial geometry of the catenary. Ultimately, this method allows the possibility of modeling catenary systems for any combination of tensile forces on the wires and pre-determined values of pre-sag. Furthermore, this method can be applied in the future to determine the undeformed catenary geometry for more complex systems including additional features such as clamps and section insulators.

The current model developed has been validated against the reference model according to the standard. It is recognized that the modelling and simulation methods outlined should be validated against real line test data in accordance to the standard. All outputs, except for the minimum uplift at the supports, satisfied the requirements from Validation 1. However, due to high stiffness values at the supports and lack of modelling line stagger, the requirements have not been met in order to begin Validation 2. 
The methodology outlined in this paper only considers a simplified model; however catenary systems are diverse and can contain different combinations of features in each span. In the future, the model can be expanded to contain additional elements such as line stagger, section insulators, and line overlaps to more closely replicate real-world networks. Detailed modeling of these features is of critical importance when understanding the real-world behaviour beyond the standard modeling requirements, as these locations typically represent the highest occurrence for loss-of-contact.

\section{ACKNOWLEDGMENT}

This research was funded by the Natural Sciences and Engineering Research Council of Canada (NSERC) and Industry Partner SNC-Lavalin. Technical advice and guidance were gratefully received from Samantha Lee, Anesh Ramkhelawon, Dion Church, Hassan Nasri, Manjodh Banwait and Richard Catlow. Their expertise was greatly appreciated.

\section{REFERENCES}

[1] Y. H. Cho, K. Lee, Y. Park, B. Kang and K.-n. Kim, "Influence of contact wire pre-sag on the dynamics of pantograph-railway catenary," Int. J. Mech. Sci., pp. 1471-1490, 2010.

[2] European Standard, Railway applications - Current collection systems Validation of simulation of the dynamic interaction between pantograph and overhead line (BS EN 50318:2002), 2002.

[3] O. Lopez-Garcia, A. Carnicero and V. Torres, "Computation of the initial equilibrium of railway overheads based on the catenary equation," Eng. Struct., vol. 28, no. 10, pp. 1387-1394, 2006.
[4] J. Benet, F. Cuartero, T. Rojo, P. Tendero and E. Arias, "Numerical algorithms for the analysis of initial configuration in a railway catenary structure and its implementation: A software tool CALPE," Math. Prob. Eng., 2015.

[5] B. W. Kim, H. G. Sung, S. Y. Hong and H. J. Jung, "Finite element nonlinear analysis for catenary structure considering elastic deformation," CMES, vol. 63, pp. 29-45, 2010.

[6] M. Tur, E. Garcia, L. Baeza and F. J. Fuenmayor, "A 3D absolute nodal coordinate finite element model to compute the initial configuration of a railway catenary," Eng. Struct., vol. 71, pp. 234-243, 2014.

[7] J. Benet, N. Cuartero, F. Cuartero, T. Rojo, P. Tendero and E. Arias, "An advanced 3D-model for the study and simulation of the pantograph catenary system," Transp. Res C, vol. 36, p. 138, 2013.

[8] Z. Liu, Y. Song, Y. Han, H. Wang, J. Zhang and Z. Han, "Advances of research on high-speed railway catenary," J. Mod. Transp, vol. 26, no. 1, pp. 1-23, 2018.

[9] K. S. Oh, R. Morin, B. Shiff, S. Roper and I. Y. Km, "An optimization method to find the initial catenary configuration by using a gradientbased algorithm," unpublished

[10] Eland Product Group, "19/2.1mm Bronze II Stranded Conductor," Eland ProductGroup,[Online].Available:https://www.elandcables.com/media/3 8133/19-2-1 mm-bronze-ii-stranded-conductor.pdf.

[11] ANSYS Inc, ANSYS Mechanical APDL Contact Technology Guide, PA: ANSYS Inc, 2017.

[12] S. Bruni, J. Ambrosio, A. Carnicero, Y. H. Cho, L. Finner, M. Ikeda, et al., "The results of the pantograph-catenary interaction benchmark," Veh. Syst. Dyn., vol 53, pp. 412-435, 2014.

[13] C. M. Pappalardo, M. Patel, B. Tinsley and A. A. Shabana, "Contact force control in multibody pantograph/catenary systems," P I Mech. Eng. K J Mul, pp. 1-22, 2015.

[14] S. Winder, Analog and Digital Filter Design, 2 ed., Elsevier, 2002. 